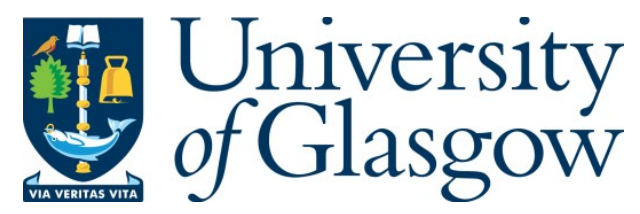

Courtial, J., Oxburgh, S., Cowie, E. N., Chaplain, G., Belin, J., Macauley, G., White, C. D., and Tyc, T. (2016) Progress towards omnidirectional transformation optics with lenses. Proceedings of SPIE, 9918, 99182V. (doi:10.1117/12.2237843).

Copyright 2016 Society of Photo-Optical Instrumentation Engineers. One print or electronic copy may be made for personal use only. Systematic reproduction and distribution, duplication of any material in this paper for a fee or for commercial purposes, or modification of the content of the paper are prohibited.

http://eprints.gla.ac.uk/129863/

Deposited on: 10 October 2016

Enlighten - Research publications by members of the University of Glasgow http://eprints.gla.ac.uk 


\title{
Progress towards omnidirectional transformation optics with lenses
}

\author{
Johannes Courtial ${ }^{\mathrm{a}}$, Stephen Oxburgh ${ }^{\mathrm{a}}$, Euan N. Cowie ${ }^{\mathrm{a}}$, Gregory Chaplain ${ }^{\mathrm{a}}$, Jakub Belin ${ }^{\mathrm{a}}$, \\ Gavin Macauley ${ }^{\mathrm{a}}$, Christopher D. White ${ }^{\mathrm{a}}$, and Tomáš Tyc ${ }^{\mathrm{b}}$

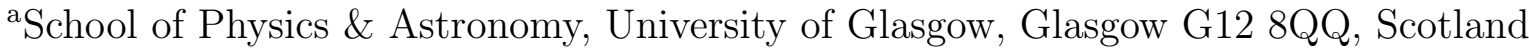 \\ binstitute of Theoretical Physics and Astrophysics, Masaryk University, Kotlarska 2, \\ 61137 Brno, Czech Republic
}

\begin{abstract}
We study, theoretically, omni-directional Euclidean transformation-optics (TO) devices comprising planar, lightray-direction changing, imaging, interfaces. We initially studied such devices in the case when the interfaces are homogeneous, showing that very general transformations between physical and electromagnetic space are possible. We are now studying the case of inhomogeneous interfaces. This case is more complex to analyse, but the inhomogeneous interfaces include ideal thin lenses, which gives rise to the hope that it might be possible to construct practical omni-directional TO devices from lenses alone. Here we report on our progress in this direction.
\end{abstract}

Keywords: transformation optics; imaging; optical design; lens systems

\section{INTRODUCTION}

Transformation optics ${ }^{1,2}$ was initially envisaged to be realised with solid metamaterial structures. The first proposed application was invisibility cloaking. The idea was that the metamaterial structure would bend any light ray around a central region; as light would therefore never reach any object placed inside that central region, the object would be invisible. Of course, the same could be achieved simply by placing the object in an opaque box, but the proposed invisibility cloak was much better than a box: by restoring light rays to their original straight-line trajectories after transmission through the cloak, the cloak itself was invisible. Better still, the design was such that the phase of light passing through the cloak was also restored to its original value, which made the cloak even more undetectable. Unfortunately, it is becoming increasingly clear that such cloak will be difficult to realise on macroscopic length scales such that it works for a wide range of visible light. ${ }^{3}$

To arrive at realisable cloaks, the original cloak design was varied in a number of ways. For example, the original cloaking idea, in which the cloak was designed to be completely invisible, was extended to include "carpet cloaks" that looked like a planar mirror, ${ }^{4}$ which can be well approximated with structures of isotropic dielectric materials ${ }^{5}$ and even just a phase hologram ("skin cloak"). ${ }^{6}$ Of most relevance to this study are designs that no longer restore the phase of transmitted light ("ray-optical cloaks"), but which still restore light rays to their original straight-line trajectories. Even then, the designs do not usually work for all light-ray directions; for example, the prism structures described in Ref. ${ }^{7}$ work around a number of directions, whereas the combinations of prisms, mirrors and lenses described in Refs. ${ }^{8}$ The same is true for the so-called "Rochester cloak", a series of four lenses that restore paraxial light rays to their original straight-line trajectories. ${ }^{9}$

Our own approach to ray-optical cloaking uses micro-structured sheets to perform the required light-ray direction changes. The sheets, called generalised confocal lenslet arrays (GCLAs), ${ }^{10}$ are arrays of micro telescopes that change the direction of transmitted light rays. Transmission through a micro telescope typically also offsets light rays, but if the offset is small, which can be achieved by making the telescopes themselves small, it can be almost unnoticeable, ${ }^{11}$ and GCLAs appear to be performing generalised refraction. In a sense, the microtelescopes act like the pixels of GCLAs, which is why we sometimes refer to the direction change they introduce

Further author information: (Send correspondence to J.C.)

J.C.: E-mail: johannes.courtial@glasgow.ac.uk, Telephone: +44 (0)141 3306429

Metamaterials, Metadevices, and Metasystems 2016, edited by Nader Engheta, Mikhail A. Noginov, Nikolay I. Zheludev, Proc. of SPIE Vol. 9918, 99182V

(C) 2016 SPIE $\cdot$ CCC code: 0277-786X/16/\$18 · doi: 10.1117/12.2237843 
as pixellated generalised refraction. The generalised law of refraction has 7 degrees of freedom ${ }^{12}$ and is general enough to include the direction changes required for very general imaging. ${ }^{13}$ Imaging is at the heart of TO, as the coordinate transformation referred to in the name maps not only positions on individual light-ray trajectories, but also light-ray intersections. The pixellation of GCLAs adds a complication: the imaging is then not stigmatic (individual light rays intersect), but integral (the axes of bundles of rays intersect). In this context, it is worth mentioning the digital cloak, ${ }^{14}$ an active cloak that uses a combination of a 3D camera and display to create an integral image of the scene behind the cloak. Back in the realm of passive cloaks, the imaging that can be achieved with GCLAs is so general that it enables omni-directional TO devices to be constructed. ${ }^{15-17}$

All our devices have the following structure. The physical space inside the device is divided into nonoverlapping tetrahedra. Such a structure is a simplicial complex (a tetrahedron is a $3 \mathrm{D}$ simplex; a 2D simplex is a triangle, a 1D simplex is a straight line). Each tetrahedron has triangular faces; planar GCLAs are placed inside each face (in faces shared between neighbouring tetrahedra, one GCLA is placed). If the GCLA parameters are chosen correctly, then any point on the inside of the physical-space simplicial complex is mapped to a corresponding position in a distorted, EM-space, simplicial complex. This means that a point object placed at any physical-space position inside the device would be seen, from the outside, in another position, namely the EM-space position corresponding to the physical-space position. It would be seen in this position irrespective of the direction from which it is viewed, and so all possible combinations of GCLAs through which light rays can travel from the physical-space position to the outside of the device must image the physical-space position to the same corresponding EM-space position. The same is true for all other physical-space positions. In other words, there exists a unique mapping from physical space to EM space.

Note that we have so far only designed our devices theoretically. The lack of a physical realisation is, at least in part, due to the shortcomings of GCLAs. ${ }^{18,19}$ Motivated by the desire to avoid these shortcomings, we are working on designing TO devices with the structure described above, but which achieve this not with GCLAs but with simple lenses. Here we describe our progress in this direction.

\section{TRANSFORMATION OPTICS WITH HOMOGENEOUS GCLAS}

The first omni-directional TO device we designed ${ }^{22}$ is easiest to understand. In this design, all GCLAs placed inside the triangular faces of the tetrahedra are homogeneous, i.e. all micro-telescopes that form particular GCLAs are displaced, but otherwise identical.

The mapping between physical space and EM space is then particularly simple. If the inside of a physical-space tetrahedron was filled with small spheres, then, in EM space, every single one of these spheres would be distorted in the same way; for example, in EM space, all spheres might be half their physical-space size. Mathematically, the space inside the physical-space tetrahedron is mapped to the corresponding EM-space tetrahedron through an affine transformation; globally, the mapping from physical to EM space is piecewise affine.

Fig. 1 shows an example of a TO device formed by homogeneous GCLAs. The device redirects any light ray that passes through it to its original straight-line trajectory, thereby making any objects seen through the device appear undistorted. Furthermore, it makes any object placed inside the central octahedron appear to be a magnification factor 0.5 of its actual size when seen from the outside. In the limit in which the magnification factor is zero, the object would be completely invisible, and the device would be an invisibility cloak as envisaged in the original proposal. ${ }^{2}$ However, as this limit usually - perhaps always - corresponds to diverging material parameters, we call a device that can be constructed from materials with non-diverging material parameters and in which the modulus of the magnification factor is less than 1 , so that objects appear smaller than they actually are, but greater than zero, so that the objects do not disappear entirely, an "honest cloak".

\section{TRANSFORMATION OPTICS WITH INHOMOGENEOUS GCLAS}

The devices described in the previous section can be generalised by allowing the GCLAs to be inhomogeneous. The telescopes that form particular inhomogeneous GCLAs can now vary across the aperture. The inhomogeneous GCLAs used inside the TO devices described here retain the property that they perform integral imaging of all space. A Gabor superlens ${ }^{23,24}$ - a confocal combination of two lenslet arrays with different periodicities — is an example for such GCLAs. Such GCLAs can be seen as pixellated, integral-imaging, analogs of glenses, 

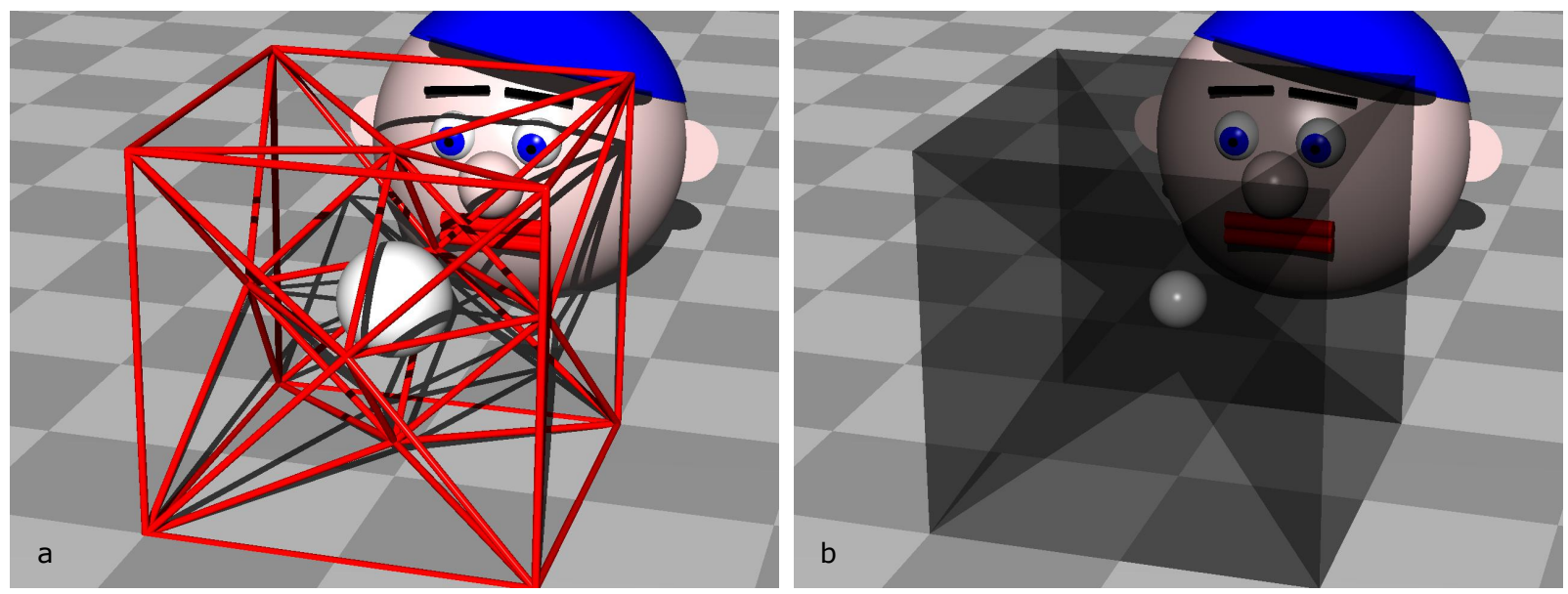

Figure 1. Octahedral "honest cloak". (a) Cylinder-frame model of the cloak, in which the edges of the GCLAs are indicated by red cylinders. Note that several of the cells that make up the cloak are not tetrahedral. This can be the case when the GCLAs that would be placed in the triangular face common to two neighbouring tetrahedra are not required, i.e. if no light-ray-direction change is required when light rays pass between the neighbouring tetrahedra. A white sphere has been placed inside the central octahedron. (b) With the cylinder frame removed and the GCLAs inserted, any object outside the cloak and seen through it appears undistorted. Any object placed inside the central octahedron, such as the white sphere, appear at a different size, in the example shown here at half of their actual size. The GCLAs are idealised in a number of ways; specifically, pixellation, field-of-view limitations (which occur in real GCLAs ${ }^{18}$ ), and other effects (such as diffraction) are neglected. To make the GCLAs visible, the transmission coefficient of each pair of GCLAs is set to 0.8 , independent of direction. Both images were calculated using the open-source scientific raytracer Dr TIM. ${ }^{20,21}$

hypothetical, planar, non-pixellated, interfaces that change the direction of transmitted light rays such that every position is imaged stigmatically. ${ }^{25}$ Glenses are idealised thin lenses, generalised to have different focal lengths on the two sides.

Compared to TO devices made from homogeneous GCLAs, TO devices made from inhomogeneous GCLAs consist of more complex GCLAs and perform more complex mappings between physical and EM space, but they also have a number of advantages. Firstly, again compared to TO devices made from homogeneous GCLAs, those made from inhomogeneous GCLAs can achieve a similar effect with fewer GCLAs. This is an important advantage, but from the perspective of this article there is a much more important advantage, related to the fact that the inhomogeneous GCLAs that form such devices are pixellated analogs of glenses, i.e. thin lenses with two different focal lengths: the devices can be designed such that a subset of the inhomogeneous GCLAs are pixellated analogs of glenses not with two different focal lengths, but with two identical focal lengths - lenses. Those lenses are significantly easier to realise than GCLAs, and they do not suffer from the shortcomings of GCLAs.

Fig. 2 shows an example of such a device, an honest cloak made from glenses. Like the honest cloak shown in Fig. 1, any object placed inside the central volume appears to be half its actual size. It can be seen that the complexity of the physical-space structure of the device, and the number of GCLAs required, is reduced in the device shown in Fig. 2 compared to that shown in Fig. 1: the device contains glenses on the six faces of an inner and on those of an outer cube, and 12 glenses that connect corresponding edges of the inner and outer cubes. The glenses connecting the edges are actually idealised lenses.

It is perhaps worth discussing the idea of adding lenses or glenses to the Rochester cloak ${ }^{9}$ in order to make it omni-directional. The Rochester cloak consists of four lenses, $\mathrm{L}_{1}$ to $\mathrm{L}_{4}$, that share a common optical axis. Fig. 3 shows these four lenses, schematically. The focal lengths of the lenses are $f_{1}$ to $f_{4}$. Lenses $\mathrm{L}_{i}$ and $\mathrm{L}_{i+1}$ are separated by $t_{i}$. With the symmetric choice $f_{4}=f_{1}, f_{3}=f_{2}$, and $t_{3}=t_{1}$, the solution for the distances is

$$
t_{1}=f_{1}+f_{2}, \quad t_{2}=2 f_{2} \frac{f_{1}+f_{2}}{f_{1}-f_{2}} .
$$



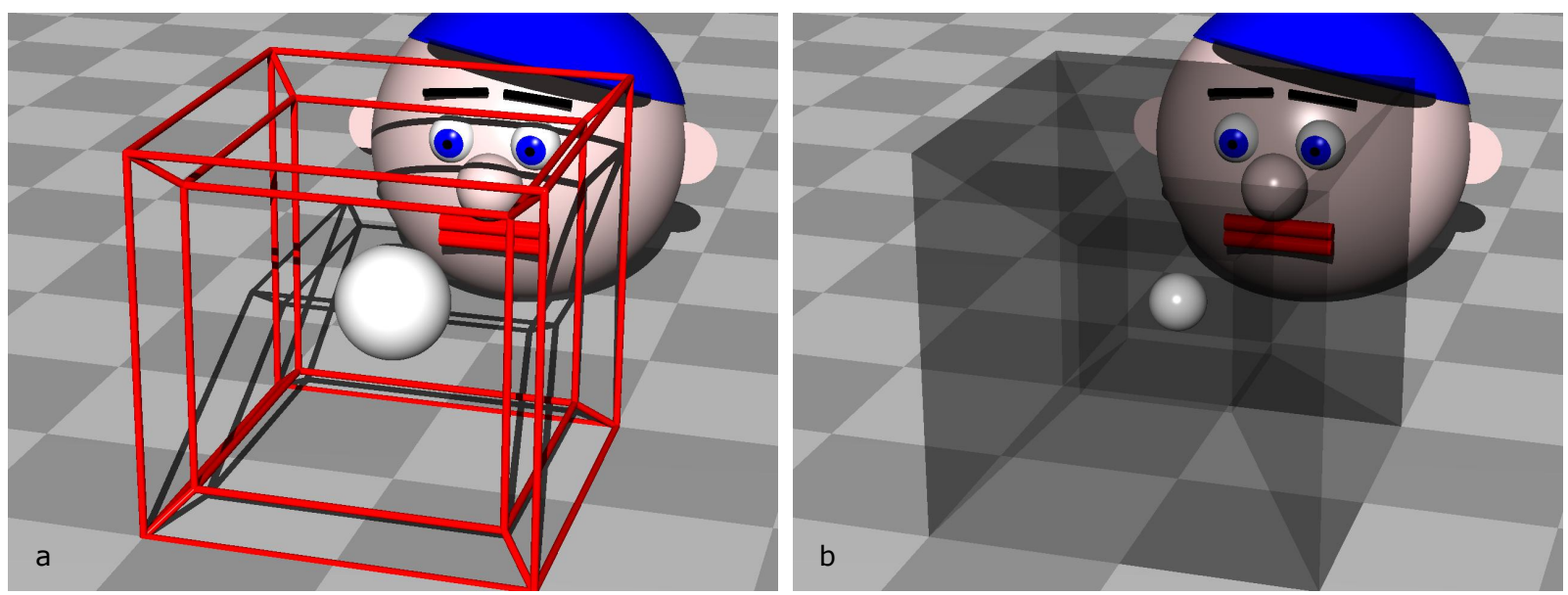

Figure 2. Glens cloak. Like in Fig. 1, a raytracing simulation of the cylinder-frame model (a) and of the actual idealised device (b) are shown.

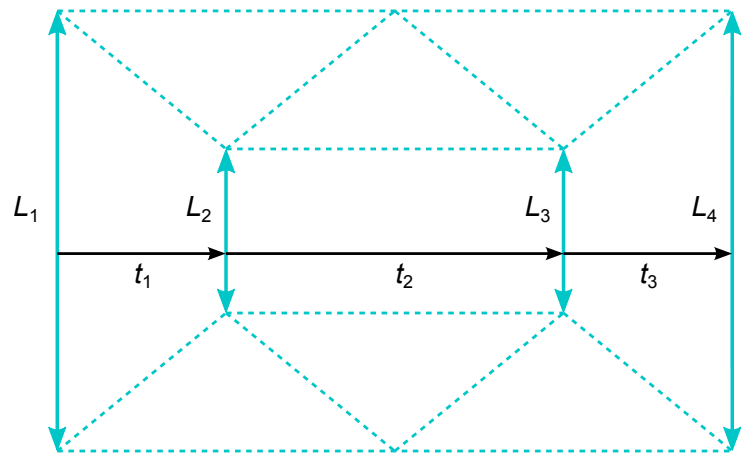

Figure 3. Example of an unsuccessful extension of the Rochester cloak. The four lenses forming the Rochester cloak are indicated as the four solid, vertical, cyan, double-sided arrows. Additional glenses aimed at making the structure an omni-directional TO device are indicated as dashed cyan lines. It can be shown that no choice of focal lengths makes the proposed structure an omni-directional TO device.

We studied several proposed designs for extended Rochester cloaks, such as the one also indicated in Fig. 3, but none of the required extensions restored all incident light rays back to their original straight-line trajectories.

\section{DISCUSSION}

TO devices based on GCLAs represent a novel compromise: on the plus side, they should large-scale, lightweight, white-light devices; on the minus side, they suffer from the imperfections of GCLAs such as limited field of view and, even within the field of view, sometimes high absorption, ${ }^{18}$ and also the less severe Fresnel losses. This new compromise opens up new possibilities, and we are actively investigating a number of applications such as an energy-saving window. ${ }^{16}$

We have described here steps in the direction of designing devices that use the same principles but in which as many of the GCLAs as possible are replaced by lenses. The devices made from inhomogeneous GCLAs, which are simpler in structure and which contain a number of lenses, are a considerable success in this direction.

\section{ACKNOWLEDGMENTS}

This work was supported by the Engineering and Physical Sciences Research Council [grant numbers EP/K503058/1 and EP/M010724/1]. T. Tyc acknowledges support of grant P201/12/G028 from Grantová Agentura České Republiky. 


\section{REFERENCES}

[1] U. Leonhardt, "Optical conformal mapping," Science 312, pp. 1777-1780, 2006.

[2] J. B. Pendry, D. Schurig, and D. R. Smith, "Controlling electromagnetic fields," Science 312, pp. 1780-1782, 2006.

[3] F. Monticone and A. Alù, "Invisibility exposed: physical bounds on passive cloaking," Optica 3, pp. 718-724, 2016.

[4] J. Li and J. B. Pendry, "Hiding under the carpet: A new strategy for cloaking," Phys. Rev. Lett. 101, p. 203901, 2008.

[5] J. Valentine, J. Li, T. Zentgraf, G. Bartal, and X. Zhang, "An optical cloak made of dielectrics," Nature Materials 8, pp. $568-571,2009$.

[6] X. Ni, Z. J. Wong, M. Mrejen, Y. Wang, and X. Zhang, "An ultrathin invisibility skin cloak for visible light," Science 349, pp. 1310-1314, 2015.

[7] H. Chen, B. Zheng, L. Shen, H. Wang, X. Zhang, N. Zheludev, and B. Zhang, "Ray-optics cloaking devices for large objects in incoherent natural light," Nature Commun. 4, p. 2652, 2013.

[8] J. C. Howell, J. B. Howell, and J. S. Choi, "Amplitude-only, passive, broadband, optical spatial cloaking of very large objects," Appl. Opt. 53, pp. 1958-1963, 2014.

[9] J. S. Choi and J. C. Howell, "Paraxial ray optics cloaking," Opt. Express 22, pp. 29465-29478, 2014.

[10] A. C. Hamilton and J. Courtial, "Generalized refraction using lenslet arrays," J. Opt. A: Pure Appl. Opt. 11, p. 065502, 2009.

[11] A. C. Hamilton and J. Courtial, "Metamaterials for light rays: ray optics without wave-optical analog in the ray-optics limit," New J. Phys. 11, p. 013042, 2009.

[12] S. Oxburgh, C. D. White, G. Antoniou, and J. Courtial, "Law of refraction for generalised confocal lenslet arrays," Opt. Commun. 313, pp. 119-122, 2014.

[13] J. Courtial, "Geometric limits to geometric optical imaging with infinite, planar, non-absorbing sheets," Opt. Commun. 282, pp. 2480-2483, 2009.

[14] J. S. Choi and J. C. Howell, "Digital integral cloaking," Optica 3, pp. 536-540, 2016.

[15] S. Oxburgh, C. D. White, G. Antoniou, E. Orife, and J. Courtial, "Transformation optics with windows," Proc. SPIE 9193, p. 91931E, 2014.

[16] S. Oxburgh, C. D. White, G. Antoniou, E. Orife, T. Sharpe, and J. Courtial, "Large-scale, white-light, transformation optics using integral imaging," J. Opt. 18, p. 044009, 2016.

[17] T. Tyc, S. Oxburgh, E. N. Cowie, G. J. Chaplain, G. Macauley, C. D. White, and J. Courtial, "Omnidirectional transformation-optics cloak made from lenses and glenses," J. Opt. Soc. Am. A 33, pp. 1032$1040,2016$.

[18] T. Maceina, G. Juzeliūnas, and J. Courtial, "Quantifying metarefraction with confocal lenslet arrays," Opt. Commun. 284, pp. 5008-5019, 2011.

[19] J. Courtial, "Standard and non-standard metarefraction with confocal lenslet arrays," Opt. Commun. 282, pp. 2634-2641, 2009.

[20] S. Oxburgh, T. Tyc, and J. Courtial, "Dr TIM: Ray-tracer TIM, with additional specialist capabilities," Comp. Phys. Commun. 185, pp. 1027-1037, 2014.

[21] D. Lambert, A. C. Hamilton, G. Constable, H. Snehanshu, S. Talati, and J. Courtial, "TIM, a ray-tracing program for METATOY research and its dissemination," Comp. Phys. Commun. 183, pp. 711-732, 2012.

[22] S. Oxburgh and J. Courtial, "Perfect imaging with planar interfaces," J. Opt. Soc. Am. A 30, pp. 2334-2338, 2013.

[23] C. Hembd-Sölner, R. F. Stevens, and M. C. Hutley, "Imaging properties of the Gabor superlens," J. Opt. A: Pure Appl. Opt. 1, pp. 94-102, 1999.

[24] D. Gabor, "Optical system composed of lenticules." U.S. Patent 2,351,034, June 13, 1944.

[25] G. J. Chaplain, G. Macauley, J. Bělín, T. Tyc, E. N. Cowie, and J. Courtial, "Ray optics of generalized lenses," J. Opt. Soc. Am. A 33, pp. 962-969, 2016. 